\title{
ON SEMIGROUPS AND GROUPS OF LOCAL POLYNOMIAL FUNCTIONS
}

\author{
WILFRIED NÖBAUER \\ (Received 14 November 1978; revised 8 January 1979) \\ Communicated by $\mathbf{R}$. Lidl
}

\begin{abstract}
Let $Z_{n}$ be the factor ring of the integers $\bmod n$ and $t$ be a positive integer. In this paper some results are given on the structure of the semigroup of all mappings from $Z_{n}$ into $Z_{n}$ and on the structure of the group of all permutations on $Z_{n}$, which, for any $t$ elements, can be represented by a polynomial function. If $n=a b$ and $a, b$ are relatively prime, then this (semi)group is isomorphic to the direct product of the respective (semi)groups for $a$ and $b$. Thus it is sufficient to consider only the case where $n=p^{e}, p$ being a prime. In this case it is proved, that the (semi)group is isomorphic to the wreath product of a certain sub(semi)group of the symmetric (semi)group on $Z_{p^{e-1}}$ by the symmetric (semi)group on $Z_{p}$. Some remarks on these sub(semi)groups are given.
\end{abstract}

Subject classification (Amer. Math. Soc. (MOS) 1970): 20 B 99, 13 B 25.

Let $M$ be a set, $\operatorname{Sym} M$ the symmetric semigroup on $M$ and $K \operatorname{sym} M$ the symmetric group on $M$. Let $U$ be a subsemigroup of $\operatorname{Sym} M$. A function $\varphi \in \operatorname{Sym} M$ is called a $t$-local $U$-function if for any (not necessarily distinct) elements $a_{1}, a_{2}, \ldots, a_{t} \in M$ there exists a function $f \in U$, such that

$$
\varphi\left(a_{i}\right)=f\left(a_{i}\right), \quad i=1,2, \ldots, t .
$$

Let $L_{l}(U)$ be the set of all $t$-local $U$-functions. As one can see easily, $L_{t}(U)$ is a subsemigroup of $\operatorname{Sym} M$. Hence the intersection $L(U)$ of all subsemigroups $L_{l}(U)$ is also a subsemigroup of $\operatorname{Sym} M$, and

$$
L_{1}(U) \supseteq L_{2}(U) \supseteq \ldots \supseteq L(U) \supseteq U .
$$

For any subsemigroup $T$ of $\operatorname{Sym} M$, we denote the intersection of $T$ and $K \operatorname{Sym} M$ by $K T$ and call this subsemigroup of $T$ the invertible kernel of $T$. Then

$$
K L_{1}(U) \supseteq K L_{2}(U) \supseteq \ldots \supseteq K L(U) \supseteq K U .
$$


If in the chain (1) two members are equal, then clearly the corresponding members in the chain (2) also are equal. If $V$ is a subsemigroup of $U$, then $L_{d}(V)$ is a subsemigroup of $L_{t}(U)$. If $M$ is finite, then $L(U)=U, K L(U)=K U$ and all members of the chain (2) are groups.

In this paper we consider the case, where $M=Z /(n)=Z_{n}$ is a factor ring of the ring $Z$ of the integers and $U=P_{1}(Z /(n))=U(n)$ is the set of all polynomial functions on $Z_{n}$. Lausch and Nöbauer (1978) have computed the 'length' of the chain of semigroups $L_{l}(U(n))$; that is, the least $t$ such that $L_{l}(U(n))=U(n)$. In this paper some results are given on the structure of the semigroups $L_{l}(U(n))$ and the groups $K L_{l}(U(n))$. Since $L_{1}(U(n))=\operatorname{Sym} Z_{n}$ and $K L_{1}(U(n))=K \operatorname{Sym} Z_{n}$, we can assume that $t \geqslant 2$.

THEOREM 1. If $n=a b$ and $a, b$ are relatively prime, then $L_{l}(U(n))$ is isomorphic to the direct product $L_{l}(U(a)) \times L_{l}(U(b))$, and $K L_{l}(U(n))$ is isomorphic to the direct product $K L_{l}(U(a)) \times K L_{l}(U(b))$.

Proof. The first assertion is a special case of Theorem 2 in Dorninger and Nöbauer (1978); but it can also be proved directly as follows:

Let $\delta$ be the canonical epimorphism from $Z_{n}$ to $Z_{a}$. We define a function $\varphi_{a} \in \operatorname{Sym} Z_{a}$ by

$$
\varphi_{a}(\delta u)=\delta \varphi(u)
$$

for any $u \in Z_{n}$, and similarly we define a function $\varphi_{b} \in \operatorname{Sym} Z_{b}$. A straightforward argument shows, that $\left(\varphi_{a}, \varphi_{b}\right) \in L_{l}(U(a)) \times L_{l}(U(b))$. It is also easy to prove, that

$$
\varphi \rightarrow\left(\varphi_{a}, \varphi_{b}\right)
$$

defines an isomorphism from $L_{l}(U(n))$ onto $L_{l}(U(a)) \times L_{l}(U(b))$.

Since, in general, $K L_{l}(U(m))$ is the set of all those elements of $L_{l}(U(m))$, which have an inverse element in $L_{l}(U(m))$, the second assertion also holds.

By Theorem 1, it is sufficient to consider only the case where $n=p^{e}, p$ being a prime and $e>0$ an integer. First we remark, that $L_{1}(U(p))=U(p)$, hence $K L_{1}(U(p))=K U(p)$.

Let $W\left(p^{e}\right)$ be the set of all functions of $\operatorname{Sym} Z_{p^{e}}$, which are of the form

$$
x \rightarrow a_{0}+a_{1} x+p a_{2} x^{2}+\ldots+p^{e-1} a_{e} x^{e},
$$

where the $a_{i}$ are given elements of $Z_{p^{\text {e }}}$. As proved in Lausch and Nöbauer (1973), Lemma $5.9, W\left(p^{e}\right)$ is a subsemigroup of $U\left(p^{e}\right)$.

THEOREM 2. If $e \geqslant 2$, then $L_{l}\left(U\left(p^{e}\right)\right)$ is isomorphic to the wreath product of $L_{l}\left(W\left(p^{e-1}\right)\right)$ by $\operatorname{Sym} Z_{p}$, and $K L_{l}\left(U\left(p^{e}\right)\right)$ is isomorphic to the wreath product of $K L_{i}\left(W\left(p^{e-1}\right)\right)$ by $K \operatorname{Sym} Z_{p}$. 
PROOF. Assume that $\varphi \in L_{l}\left(U\left(p^{e}\right)\right)$ and that $0 \leqslant a<p, 0 \leqslant x<p^{e-1}$ then

$$
\varphi(a+p x)=c_{a}+p \psi_{a}(x),
$$

where $0 \leqslant c_{a}<p$ and $\psi_{a} \in \operatorname{Sym} Z_{p^{e-1}}$. Given $x_{1}, x_{2}, \ldots, x_{l}$, then there exists $f \in U\left(p^{e}\right)$ such that for all $x_{i}$

$$
\begin{aligned}
\varphi\left(a+p x_{i}\right) & =f\left(a+p x_{i}\right)=f(a)+f^{\prime}(a) p x_{i}+\frac{1}{2} f^{\prime \prime}(a) p^{2} x_{i}^{2}+\ldots= \\
& =f(a)+p\left(f^{\prime}(a) x_{i}+p \frac{1}{2} f^{\prime \prime}(a) x_{i}^{2}+\ldots\right),
\end{aligned}
$$

which shows that $\psi_{a} \in L_{d}\left(W\left(p^{e-1}\right)\right)$.

Conversely, given $\varphi \in \operatorname{Sym} Z_{p^{*}}$ such that

$$
\varphi(a+p x)=c_{a}+p \psi_{a}(x), \quad 0 \leqslant a<p, \quad 0 \leqslant x<p^{e-1}, \quad \psi_{a} \in L_{l}\left(W\left(p^{e-1}\right)\right)
$$

then, by Lausch and Nöbauer (1973), Proposition 5.61, $\varphi \in L_{l}\left(U\left(p^{e}\right)\right)$.

Let $\rho \in \operatorname{Sym} Z_{p}$ be defined by $\rho a=c_{a}, a=0,1, \ldots, p-1$. Then

$$
\varphi \rightarrow\left(\rho ; \psi_{0}, \psi_{1}, \ldots, \psi_{p-1}\right)
$$

defines a bijection from $L_{l}\left(U\left(p^{e}\right)\right)$ onto the set $\operatorname{Sym} Z_{p} \times L_{l}\left(W\left(p^{e-1}\right)\right)^{p}$.

Suppose that under the above bijection the element $\psi \in L_{l}\left(U\left(p^{e}\right)\right)$ is mapped onto $\left(\sigma ; \chi_{0}, \chi_{1}, \ldots, \chi_{p-1}\right)$, then

$$
\varphi \psi(a+p x)=\varphi\left(\sigma a+p \chi_{a}(x)\right)=\rho \sigma a+p \psi_{\sigma a} \chi_{a}(x),
$$

hence

$$
\varphi \psi \rightarrow\left(\rho \sigma ; \psi_{\sigma 0} \chi_{0}, \psi_{\sigma 1} \chi_{1}, \ldots, \psi_{\sigma(p-1)} \chi_{(p-1)}\right) .
$$

This proves the first assertion. Since $\varphi$ is a permutation if and only if $\rho$ and all $\psi_{i}$ are permutations, the second assertion is also true.

REMARK. It is well known, that $U\left(p^{e}\right)$ is isomorphic to the wreath product of $W\left(p^{e-1}\right)$ by $\operatorname{Sym} Z_{p}$ and that $K U\left(p^{e}\right)$ is isomorphic to the wreath product of $K W\left(p^{e-1}\right)$ by $K \operatorname{Sym} Z_{p}$.

COROLLARY. For any $e \geqslant 1, L_{l}\left(W\left(p^{e}\right)\right)=L_{t+1}\left(W\left(p^{e}\right)\right)$ if and only if

$$
L_{l}\left(U\left(p^{e+1}\right)\right)=L_{t+1}\left(U\left(p^{e+1}\right)\right),
$$

and $L_{l}\left(W\left(p^{e}\right)\right)=W\left(p^{e}\right)$ if and only if $L_{l}\left(U\left(p^{e+1}\right)\right)=U\left(p^{e+1}\right) . A$ result of the same kind is true for the invertible kernels of these semigroups.

From the results of Lausch and Nöbauer (1978) we now easily can obtain the length of the chain of the semigroups $L_{l}\left(W\left(p^{e}\right)\right)$, and moreover we can see that there is no equality within this chain. 
REMARK. $L_{2}\left(W\left(p^{e}\right)\right)=L_{2}\left(U\left(p^{e}\right)\right)$ and $K L_{2}\left(W\left(p^{e}\right)\right)=K L_{2}\left(U\left(p^{e}\right)\right)$.

Proof. We have only to prove the first statement. Clearly $L_{2}\left(W\left(p^{e}\right)\right) \subseteq L_{2}\left(U\left(p^{e}\right)\right)$. Conversely suppose $\varphi \in L_{2}\left(U\left(p^{e}\right)\right)$; then $\varphi$ is a compatible function on $Z_{p^{e}}$-that means, for any congruence relation $\theta$ on $Z_{p^{e}}, u \equiv v \bmod \theta \operatorname{implies} \varphi(u) \equiv \varphi(v) \bmod \theta$. Taking for $\theta$ the congruence relation corresponding to the principal ideal of $Z_{p}$, which is generated by $b-a$, we see that $\varphi(b)-\varphi(a)=r(b-a)$. Thus

$$
l(x)=\varphi(a)+r(x-a)
$$

is a polynomial, such that $l(a)=\varphi(a), l(b)=\varphi(b)$. Since $l(x) \in W\left(p^{e}\right)$, we now see that $\varphi \in L_{2}\left(W\left(p^{e}\right)\right)$, which completes the proof.

Finally, we consider the length of the chain of the invertible kernels of the chain of the semigroups $L_{l}\left(U\left(p^{e}\right)\right)$.

LEMMA. Let $e \geqslant 2$ and $f \neq 2$ be a natural number, such that $L_{f}\left(U\left(p^{e}\right)\right) \supset L_{f+1}\left(U\left(p^{e}\right)\right)$. Then also $K L_{f}\left(U\left(p^{e}\right)\right) \supset K L_{f+1}\left(U\left(p^{e}\right)\right)$.

Proof. Since there exist permutations of $Z_{p^{e}}$ which are not compatible, our statement holds for $f=1$. Suppose that $f>2$ and $L_{f}\left(U\left(p^{e}\right)\right) \supset L_{f+1}\left(U\left(p^{e}\right)\right)$. By Lausch and Nöbauer (1978), Theorem 3 and Theorem 4, then $f+\varepsilon(f) \leqslant e$, where $\varepsilon(f)$ is the exponent of the greatest power of $p$ which divides $f$. Let us consider the function $\pi \in \operatorname{Sym} Z_{p^{e}}$ defined by

$$
\begin{aligned}
\pi(a+p x) & =(a+p x)+p^{f-1} x(x-1) \ldots(x-(f-1)), \\
a & =0,1, \ldots, p-1, \quad x=0,1, \ldots, p^{e-1} .
\end{aligned}
$$

This function is a permutation of $Z_{p^{e}}$, since

$$
\pi(a+p x)=a+p\left(x+p^{f-2} x(x-1) \ldots(x-(f-1))\right.
$$

and the function $g \in \operatorname{Sym} Z_{p^{s-1}}$, defined by

$$
g(x)=x+p^{f-2} x(x-1) \ldots(x-(f-1)),
$$

is a permutation of $Z_{p^{e-1}}$, which follows by Lausch and Nöbauer (1973), Proposition 4.31 (since $g^{\prime}(x) \equiv 1 \bmod p$ for all $x$ ). By Lausch and Nöbauer (1978), $\pi \in L_{f}\left(U\left(p^{e}\right)\right)$ but $\pi \notin L_{f+1}\left(U\left(p^{e}\right)\right)$.

THEOREM 3. The length of the chain of the groups $K L_{t}\left(U\left(p^{e}\right)\right)$ equals the length of the chain of the semigroups $L_{l}\left(U\left(p^{e}\right)\right)$, unless $p^{e}=2^{3}$ or $3^{2}$, in which cases the length of the first chain is one less than the length of the second chain. 
Proof. By our lemma, we have only to consider the case, where the length of the second chain equals 3 . If, in this case, $K L_{2}\left(U\left(p^{e}\right)\right)=K U\left(p^{e}\right)$, then every compatible permutation is a polynomial function, since, by the proof of the remark, every compatible function on $Z_{p^{*}}$ is in $L_{2}\left(U\left(p^{e}\right)\right)$. But, by our hypothesis on the length of the first chain, not every compatible function is a polynomial function. Thus $Z_{p^{e}}$ is 1-permutation-hemiprimal, but not 1-hemiprimal in the sense of Nöbauer (1974). In Corollary 6.4 of that paper (by comparing the number of all compatible permutations on $Z_{p}$ with the number of all polynomial functions on $p^{e}$ which are permutations), it has been proved, that $Z_{p^{*}}$ is 1 -permutation hemiprimal, but not 1-hemiprimal, if and only if $p^{e}=2^{3}$ or $3^{2}$.

\section{References}

D. Dorninger and W. Nöbauer (1979), "Local polynomial functions on lattices and universal algebras', Colloq. Math., to appear.

H. Lausch and W. Nöbauer (1973), Algebra of polynomials (North-Holland, AmsterdamLondon).

H. Lausch and W. Nöbauer (1979), 'Local polynomial functions on factor rings of the integers', J. Austral. Math. Soc., to appear.

W. Nöbauer (1974), 'Compatible and conservative functions on residue-class rings of the integers', Coll. Math. Soc. János Bolyai 13, Topics in number theory, 245-257.

Institut für Algebra und

Mathematische Strukturtheorie

Technische Universität

Argentinierstrasse 8

A-1040 Wien

Austria 